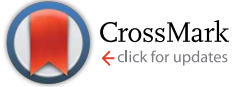

Cite this: RSC Adv., 2017, 7, 14631

Received 16th January 2017

Accepted 27th February 2017

DOI: $10.1039 / c 7 r a 00654 c$

rsc.li/rsc-advances

\section{Multicompartment-like aggregates formed by a redox-responsive surfactant encapsulated polyoxometalate in DMF/butanol mixed solvent $\uparrow$}

\begin{abstract}
Na Li, Sijing Yi, Zhaohong Qian, Jiao Wang, Nana Lei and Xiao Chen*
The unique and interesting aggregation behaviors of a novel surfactant-encapsulated complex (SEC), composed of a Keggin-type polyoxometalate (POM), phosphotungstic acid and a redox-responsive ferrocene-containing cationic surfactant, dimethyldioctadecylammonium bromide (BFDMA), have been investigated in $\mathrm{N}, \mathrm{N}$-dimethylformamide (DMF)/butanol mixed solvent. By carefully tuning the composition of the solvent, hollow spherical aggregates with multilayered shells were initially observed with a diameter of $55 \pm 9 \mathrm{~nm}$ using transmission or scanning electron microscopes. Owing to the solvophobic effect and $\pi-\pi$ stacking interaction between alkyl chains or ferrocene groups, these spherical aggregates were found to gradually fuse together into aggregates with flower-like and finally compartment-like morphologies with an average size of 120-150 nm. Based on X-ray diffraction measurements and the molecular configuration data of SEC, the multilayers in aggregates were composed of bilayers of SEC with the POM inside and three rearranged BFDMA molecules outside. The morphology of such aggregates was partially reversible under electrochemical modulation. Applying an anodic oxidation potential would induce the disassembling of compartment-like aggregates into irregular spheres with a size of about $200 \mathrm{~nm}$, which could be turned back after applying a cathodic reduction potential. The obtained results here should shed light on the design of novel nanostructures from such inorganic/organic hybrid clusters.
\end{abstract}

\section{Introduction}

A variety of stimuli-responsive amphiphilic molecules with particular physical and chemical properties have been designed by bridging a series of functional units including unsaturated units, chiral groups, stimuli-responsive groups and so on. ${ }^{1-3}$ Their self-assemblies formed in solution have exhibited attractive application potentials for photorheological fluids, novel materials and drug delivery. ${ }^{4-6}$ By a supra-molecular strategy, through various noncovalent interactions like electrostatic or charge-transfer interactions, hydrogen bonding, van der Waals forces, $\pi-\pi$ stacking, etc., the assemblies could be imparted with characteristics of versatility, flexibility, and reversibility. ${ }^{7-9}$ Among them, surfactants containing a ferrocene group have been frequently used due to their excellent redox-responsive character and perfect processability, which allow them to be widely employed in electrochemistry, liquid-crystalline

Key Laboratory of Colloid and Interface Chemistry, Shandong University, Ministry of Education, Jinan, 250100, China. E-mail: xchen@sdu.edu.cn; Fax: +86-53188564464; Tel: +86-531-88365420

$\dagger$ Electronic supplementary information (ESI) available: The sample appearance pictures of BFDMA and SEC, and more structure information of BFDMA and SEC were characterized by elemental analysis, FTIR and TEM instrument with the results. See DOI: 10.1039/c7ra00654c materials, and sensing. ${ }^{\mathbf{1 0 - 1 3}}$ Through an anodic electrochemical stimulus, the ferrocene group can be oxidized to a hydrophilic ferrocenium cation. Switching the oxidized and reduced states of the ferrocene group may help tune both the amphiphilicity and also the charges of surfactants. ${ }^{14}$

Polyoxometalates (POMs) are clusters formed by transition metals in their highest oxidation state linked together by share oxygen atoms to form discrete anionic metal-oxide nanoclusters. $^{15,16}$ They are becoming intriguing building blocks for functional materials owing to their well-defined nanostructures and excellent physicochemical nature like conducting, magnetic, medicine and catalytic properties. ${ }^{17-20}$ Meanwhile, as negatively charged building units, the POM clusters can be encapsulated by appropriate counterions, such as the cationic surfactant, to form organic-inorganic hybrids, which could be used to provide new paths to prepare novel nanoarchitectures. ${ }^{21,22}$ For examples, the onion-like multilayered aggregates of surfactant encapsulated complex (SEC) of POM have been reported by $\mathrm{Wu}$ and his coworkers by controlling the solvent polarity. ${ }^{23}$ Similarly, Wang et $a .^{\mathbf{2 4 , 2 5}}$ have also obtained SEC aggregates with various morphologies like disk, cone, tube and sphere through controlling the POM surrounding conditions and choosing the appropriate solvent systems. By covalently grafting different alkyl chains onto the Mn-Anderson type POM cluster, Cronin et al. have obtained sea urchin-like hydrophobic assemblies. Some 
interesting physical properties like the gel to liquid-crystalline phase transition were observed. Meanwhile, Cronin and Liu's groups reported the vesicle-like aggregates formation from a $\mathrm{C}_{16}$ surfactant encapsulated POM hybrid with the hydrophilic POM facing the outside surface while the hydrophobic $\mathrm{C}_{16}$ alkyl chains towards inside. Then, Liu et al. observed the reverse-vesicle like aggregates through manipulating the solvent polarity. ${ }^{\mathbf{2 6 - 2 8}}$ Besides, Kurth and Faul et al. have managed to use the europiumexchanged derivative of POM as well as the surfactants with single, double, or even three chains to fabricate hybrid functional materials by ionic self-assembly route. The interactions between functional groups in surfactants with POM could influence the site symmetry of $\mathrm{Eu}^{3+}$ in different ways. ${ }^{29}$ In addition, a variety of research works related to the self-assembling of SEC into other different morphologies have been reported such as LangmuirBlodgett films, ${ }^{30}$ rose flower or snow flower-like aggregates. ${ }^{31}$ The fabrication of stimuli-responsive SEC self-assemblies has also attracted much attention. By covalently grafting the azobenzene (Azo) group onto the Mn-Anderson-type cluster, $\mathrm{Wu}$ et al. obtained a novel photo-responsive SEC aggregates with a fiberlike morphology in Azo trans state and a spherical structure upon UV irradiation. Then, by covalently grafting the redox-responsive ferrocene group to the Anderson-type POM, the SEC exhibited amphiphilicity and redox-responsibility, which made them selfassemble into spherical aggregates in organic solvents and changed by a redox modulation. ${ }^{32,33}$ It is interesting to find that most redox SECs were constructed through a donor-acceptor interactions to prepare charge-transfer salts. ${ }^{34,35}$ Otherwise, the ferrocene groups were covalently bonded with POM clusters. ${ }^{36}$ However, we have not found the preparation of SEC directly by using ferrocene-containing surfactant through weak noncovalent interactions.

For this motivation, we have synthesized a redox-responsive ferrocene-containing cationic surfactant, ${ }^{37}$ dimethyldioctadecylammonium bromide (BFDMA) with its chemical structure shown in Fig. 1. Through the electrostatic interaction, BFDMA could encapsulate a polyoxometalate with a Keggin-structure, phosphotungstic acid $\left(\mathrm{H}_{3}\left[\mathrm{PW}_{12} \mathrm{O}_{40}\right]\right)$, to form SEC. With the synergetic effects of solvophobic and $\pi-\pi$ stacking interactions, the aggregate with compartment-like morphology was finally produced in the mixed organic solvent, DMF/butanol. Such aggregates exhibited the redox-responsive property and could be disassembled into irregular spheres through electrochemical triggering. However, upon applying a reduction potential, the compartment-like aggregates with multilayered shells could be

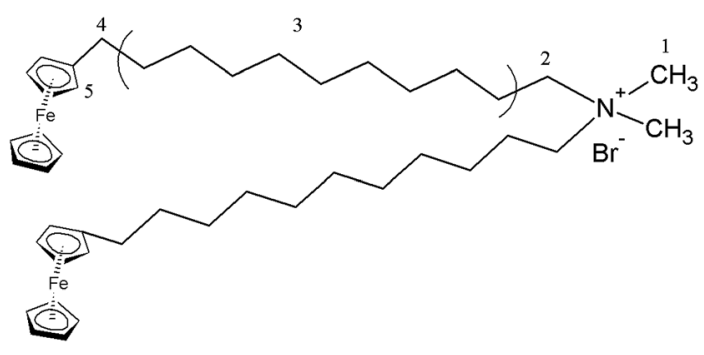

Fig. 1 Chemical structure of BFDMA. restored. Such redox-responsive aggregate might be considered as a reversibly smart host to tune the guests loading and releasing through electrochemical method.

\section{Experimental}

\subsection{Materials}

Ferrocene (99\%), 11-bromoundecanoic acid (98\%), oxalyl chloride (99\%), phosphotungstic acid $\left(\mathrm{H}_{3}\left[\mathrm{PW}_{12} \mathrm{O}_{40}\right]\right)$, chloroform (99\%), $N, N$-dimethylformamide (DMF) (99.5\%), tetrahydrofuran (THF) (99\%), methanol (99.5\%), butanol (99\%), dimethylamine solution (40 wt\%), tetrabutylammonium perchlorate (TBAP, 99\%) were obtained from Aladdin Chemistry Co., Ltd. Other chemicals were of analytical grade. All of them were used as received without further purifications. High-purity water with resistivity of $18.4 \mathrm{M} \Omega \mathrm{cm}$ was obtained from a FLOM water purification system (Qingdao). The cationic surfactant BFDMA was synthesized according to the procedures from literature. ${ }^{37}$

\subsection{Solid SEC preparation}

A weighed amount of $\mathrm{H}_{3} \mathrm{PW}_{12} \mathrm{O}_{40}(0.48 \mathrm{~g}, 0.166 \mathrm{mmol})$ was firstly dissolved in water. Then this solution was added drop by drop into the aqueous solution, in which $0.40 \mathrm{~g}(0.498 \mathrm{mmol})$ BFDMA have been dissolved, to gain the suspension of SEC composite. The initial molar ratio between BFDMA and $\mathrm{H}_{3} \mathrm{PW}_{12} \mathrm{O}_{40}$ was kept as $3: 1$. The suspension was vigorously stirred for $12 \mathrm{~h}$. Then, the solid product was filtered and washed respectively with ethanol and water of high purity in turn for several times until the filtrate was transparent, indicating the fully elimination of the un-reacted POM and BFDMA. Finally, the obtained grayish-green SEC was put in a vacuum desiccator at $50{ }^{\circ} \mathrm{C}$ until the weight of SEC kept constant. The ${ }^{1} \mathrm{H}$ NMR, elemental analysis measurements and FT-IR spectroscopy were performed to characterize the SEC composition. For SEC: ${ }^{1} \mathrm{H}$ NMR (300 MHz, DMSO-d $\left.\mathrm{d}_{6}, \delta, \mathrm{ppm}\right): 4.29-3.80(\mathrm{~m}, 18 \mathrm{H}$, $\left.\mathrm{C}_{10} \mathrm{H}_{9} \mathrm{Fe}\right), 3.20\left(\mathrm{~s}, 4 \mathrm{H}, \mathrm{CH}_{2} \mathrm{~N}\right), 2.85\left(\mathrm{~s}, 6 \mathrm{H}, \mathrm{CH}_{3} \mathrm{~N}\right), 2.43-2.14(\mathrm{~m}$, $\left.4 \mathrm{H}, \mathrm{CH}_{2} \mathrm{Fc}\right), 1.78-1.10\left(\mathrm{~m}, 36 \mathrm{H}, \mathrm{CH}_{2}\right)$.

\subsection{SEC aggregate sample preparation}

To prepare the self-assembly of SEC, it was first dissolved in its good solvent, such as $N, N$-dimethylformamide (DMF). Then, this transparent solution was dropped into a kind of poor solvent for SEC like butanol to induce the aggregation and the final concentration of SEC was $0.53 \mathrm{mg} \mathrm{mL}^{-1}$. Several combinations of good and poor solvents were investigated including tetrahydrofuran/methanol and chloroform/methanol systems. All samples in this work were prepared and annealed at $25{ }^{\circ} \mathrm{C}$.

\subsection{Characterization}

The morphology, size and other fine structures of SEC supramolecular aggregates were characterized by using a Hitachi 100CX-II transmission electron microscope (TEM) operating at $100 \mathrm{kV}$ and HRTEM (JEOL-2010) with an accelerating voltage of $200 \mathrm{kV}$. The samples were prepared on a carbon film coated copper grid by a dip coating technique. The excess sample solution was removed with a filter paper. 
The scanning electron microscopy (SEM) investigations were performed on a JSM-6700F SEM system (operated at $3.0 \mathrm{kV}$ ). To gain the three-dimensional morphology of the aggregates, the sample solution was dropped onto a clean silicon wafer and drying for $24 \mathrm{~h}$. Before examination, the dried sample was coated with a thin layer of gold.

To character the precise structure of products and the electrostatic interaction between BFDMA and POM, ${ }^{1} \mathrm{H}$ nuclear magnetic resonance $\left({ }^{1} \mathrm{H}\right.$ NMR $)$ spectra were measured using the tetramethylsilane as an internal reference at $25^{\circ} \mathrm{C}$ and recorded on a Bruker AV-300 instrument. The deuterated dimenthylsulfoxide (DMSO) was chosen as the solvent.

The Fourier transformed infrared spectroscopy (FT-IR) spectra from 400 to $3500 \mathrm{~cm}^{-1}$ that were measured by an Alpha-T spectrometer (Bruker) with a resolution of $4 \mathrm{~cm}^{-1}$ to character the effect of electrostatic interaction. Before examination, the solid sample was mixed with $\mathrm{KBr}$ powder and pressed into thin disks.

$\mathrm{UV} /$ vis spectra were measured in a quartz cell by using a U4100 instrument (Hitachi, Japan) to gain possible stacking manner of ferrocene rings and verify the oxidation of ferrocene groups. The length of the light path is $1 \mathrm{~cm}$.

To obtain precise layer structure of aggregates, X-ray diffraction (XRD) patterns of dried SECs solid samples with particular shapes were obtained by a Rigaku D/max-2200/PC diffractometer with the tube voltage and current of $40 \mathrm{kV}$ and $35 \mathrm{~mA}$ for $\mathrm{Cu} \mathrm{K} \alpha$ radiation $(\lambda=0.15418 \mathrm{~nm})$ at a scan rate of $10^{\circ} \mathrm{min}^{-1}$.

The atomic force microscopy (AFM) images for the aggregated SECs were obtained in the tapping mode under ambient conditions by a Nanoscope IIIA from Digital Instruments. As for the preparation of the sample, a few drops of sample solution were placed on a freshly cleared silicon surface. After adsorption for $24 \mathrm{~h}$, excess solution was removed by a filter paper.

The cyclic voltammetry (CV) measurements were performed in a single compartment cell at room temperature with a threeelectrode configuration using a $\mathrm{CHI}$ 650A electrochemical workstation. The $\mathrm{Ag} / \mathrm{AgCl}$ electrode, a platinum plate and a platinum wire were respectively used as the reference, working and counter electrodes. The oxidation or reduction for aggregate transition was conducted under potentiostatic manner for $4 \mathrm{~h}$.

\section{Results and discussion}

\subsection{Structural characterization for SECs}

The building block (SEC) for self-assembly here was prepared from a Keggin-type polyoxometalate $\left(\mathrm{H}_{3} \mathrm{PW}_{12} \mathrm{O}_{40}, \mathrm{POM}\right)$ and a ferrocene-containing cationic surfactant (BFDMA) by the electrostatic interaction. The obtained SEC was found not to dissolve in water, but soluble in organic solvents such as chloroform, THF and DMF. Therefore, the hydrophilic surface of POM should have been covered by the hydrophobic alkyl chains from BFDMA, which also accompanied with a color change from yellow (BFDMA) to greyish-green (SEC) as shown in Fig. S1. $\dagger$ To have more definitely and quantitatively information on SEC structure, the ${ }^{1} \mathrm{H}$ NMR and FT-IR spectra for SEC and individual BFDMA or POM have been measured with the results shown in Fig. 2.

As can be seen from the NMR spectra shown in Fig. 2a, the resonance signals between SEC and BFDMA were similar to some extent. The characteristic hydrogen signals of ferrocene group ( $\delta=4.0-4.1 \mathrm{ppm})$ and alkyl chains from BFDMA (as indicated by the atom number shown in Fig. 1) were also clearly appeared in that from SEC, indicating that the integrated structure of BFDMA was remained during the encapsulation process. However, for SEC, the proton peaks of methyl and methylene groups of BFDMA ( 1 and 2 in Fig. 2a) were noted to slightly shift to low field for about 0.08 and $0.32 \mathrm{ppm}$ compared to those from free BFDMA, respectively. This phenomenon arises from the electrostatic interaction between the ammonium group of BFDMA and $\left[\mathrm{PW}_{12} \mathrm{O}_{40}\right]^{3-}$. The immobilization of surfactant molecules on the surface of POM would reduce their mobility and cause the decrease of electron density around the $\mathrm{N}^{-\mathrm{CH}_{3}}$ and N$\mathrm{CH}_{2}$ groups. ${ }^{38-40}$

The existence and keeping of Keggin structure of POM in SEC could be certified from the characteristic vibration bands belonged to pure POM in FTIR spectrum of SEC shown in Fig. 1b. The four prominent absorption bands at 1080, 985, 889 and $806 \mathrm{~cm}^{-1}$ due to asymmetric stretchings of stretching $\mathrm{P}-\mathrm{O}, \mathrm{W}-\mathrm{O}_{\text {terminal }}, \mathrm{W}-\mathrm{O}_{\mathrm{b}}-\mathrm{W}$ interbridge between corner-sharing $\mathrm{WO}_{6}$ octahedra and $\mathrm{W}-\mathrm{O}_{\mathrm{c}}-\mathrm{W}$ interbridge between edge-sharing $\mathrm{WO}_{6}$ octahedra ${ }^{41}$ were reproduced, which confirmed the well retaining of POM in SEC
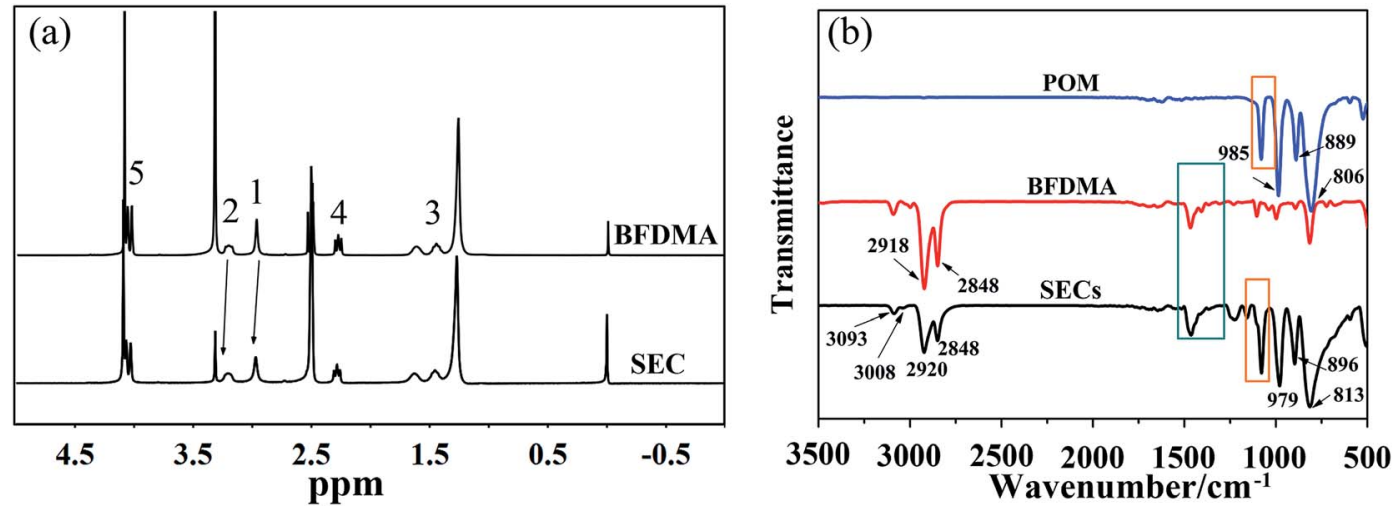

Fig. $2{ }^{1} \mathrm{H}$ NMR spectra of BFDMA and SEC (a) and FT-IR spectra of POM, BFDMA and SEC (b). 
complexes. ${ }^{40}$ However, the $\mathrm{P}-\mathrm{O}$ band at $1080 \mathrm{~cm}^{-1}$ split into 1107 and $1078 \mathrm{~cm}^{-1}$ (green frame in Fig. $2 \mathrm{~b}$ and $\mathrm{S} 2 \dagger$ ) due to the electrostatic interaction between the terminal oxygen of POM and amino group of BFDMA, which also caused shifts of other three bands. ${ }^{42}$ The bands occurred at 2920 and $2848 \mathrm{~cm}^{-1}$ in SEC came from the anti- and symmetric stretching modes respectively of methylene group from the capped BFDMA. As well known, the frequencies of the $\mathrm{CH}_{2}$ stretching bands are usually sensitive to the conformation of a hydrocarbon chains. Low frequencies of the bands are characteristic of a highly ordered alkyl chain, i.e., gauche conformers, in the hydrocarbon chain. ${ }^{43-45}$ Therefore, referring to those from the crystalline BFDMA result (2918 and $2848 \mathrm{~cm}^{-1}$ in Fig. 2b), the alkyl chains in the solid-state SEC were basically in a well-ordered trans-zigzag conformation with their molecular planes parallel to each other. The band at $1463 \mathrm{~cm}^{-1}$ shown in magnified FTIR spectrum (orange frame in Fig. $2 \mathrm{~b}$ and $\mathrm{S} 2 \dagger$ ) was from a $\mathrm{CH}_{2}$ scissoring mode SEC. The little shoulder peak at 1479 $\mathrm{cm}^{-1}$ might belong to the interaction of the $\mathrm{CH}_{2}$ groups with $\mathrm{N}^{+}$, which resulted in a charge distribution change of the carbon linked to $\mathrm{N}$ atom of SEC. ${ }^{46}$ Meanwhile, the characteristic vibration bands of $\mathrm{CH}=\mathrm{CH}$ from ferrocene group were also presented respectively at 3008 and $3093 \mathrm{~cm}^{-1}$ reflecting their well keeping at the chain terminal in SEC. ${ }^{41}$ Based on above analyses and the result of elemental analysis (see ESI $\dagger$ ), the final chemical formula of SEC could be described as (BFDMA) $)_{3} \mathrm{PW}_{12} \mathrm{O}_{40}$, where the inorganic block (POM) was capped by three cationic surfactant molecules on its surface through electrostatic interaction.

\subsection{The self-assembling behavior of SEC}

The coexistence of hydrophobic alkyl chains and hydrophilic POM clusters in SECs could make them amphiphilic and capable of self-assembling into onion-like or vesicle-like aggregates both in organic solvent and in solvent-free dried state. ${ }^{23}$ However, in the solution with only a single solvent, though the aggregate formation has been confirmed by optical microscopy and dynamic light scattering measurements, it is often impossible to get them stable in dried state due to fusion into layered structures during solvent evaporation. Usually, the addition of another stabilizing agent, like methanol or butanol, can further enhance the stability of SEC aggregates in solventfree state because these small polar molecules can improve the rigidity and the solvophobic interaction between alkyl chains. $^{24,25}$ For these reasons, in our study here, we have developed a mixed solvent system of DMF/butanol at an optimized volume mixing ratio of $3: 7$ to construct stable SEC aggregates both in solution and in dried state.

As shown in Fig. 3 for TEM images of self-assembled SEC aggregates in DMF/butanol mixed solvent at different growth time, the nearly spherical vesicle-like aggregates with hollow interior and average size of $55 \pm 9 \mathrm{~nm}$ were initially formed after dispersing SEC for about 40 minutes (Fig. 3a and S3a $\dagger$ ), and the diameter of the hollow interior was $20-30 \mathrm{~nm}$. The enlarged image shown in inset of Fig. 3a indicated the multilayered structure of walls. These spherical aggregates have a strong tendency to fuse with each other at longer growth time. With the aggregation time extending to $1 \mathrm{~h}$, the aggregate morphology was
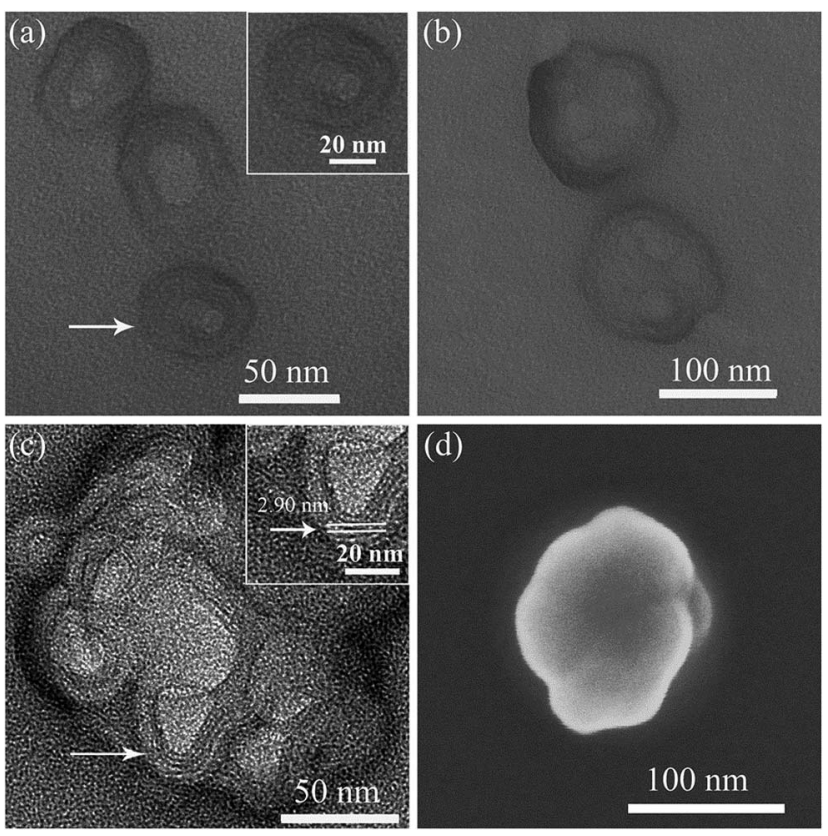

Fig. 3 TEM images of self-assembled SEC aggregates in DMF/butanol (3:7 v/v) at different growth time. (a) Spherical aggregates with multilayered shells at 40 min with the magnified image at inset; (b) multicompartment-like aggregates after $1 \mathrm{~h}$ growth with their HR-TEM (c) and SEM (d) images.

gradually evolved from the single vesicles-like to multicompartment ones (Fig. 3b and S3b $\dagger$ ) with an average size of about $120 \mathrm{~nm}$ for the later. Meanwhile, as can be seen from Fig. 3b, these multicompartment-like aggregates present anomalous shapes, different from those with regular structures reported before. ${ }^{47,48}$ It was found that such a multicompartment-like morphology was extremely stable and shape-persistent even for days or months. Fig. 3c exhibits the high-resolution TEM observation result of these multicompartment-like aggregates. It was interesting to disclose that these aggregates were composed of several small vesicle-like sphericals with multilayered shells, which similar to those formed at the initial growth stage. From Fig. $3 c$ and inset, it could be also found that the hollow interiors of small vesicle-like spheres crossed over each other, but they were separated by uniform multilayered walls with a layer spacing of $2.90 \mathrm{~nm}$. In addition, the SEM image of these aggregates shown in Fig. 3d also clearly indicated a three-dimensional morphology of mixed small spherical components, indicating the fusion induced formation of the multicompartment-like aggregates.

To give further structure information on such aggregates, the AFM and XRD measurements were also used. Fig. 4a presents the AFM image on the morphology of multicompartment-like aggregate sample. Most of them were irregular spherical structures like those observed by SEM. From the section analysis profile shown in Fig. 4b, the diameter and height were measured around $174 \mathrm{~nm}$ and $12.3 \mathrm{~nm}$, respectively. The former was a little larger than those observed by TEM. This might be resulted from the presence of un-assembled SECs, which might combine or deposit onto the already assembled aggregates 

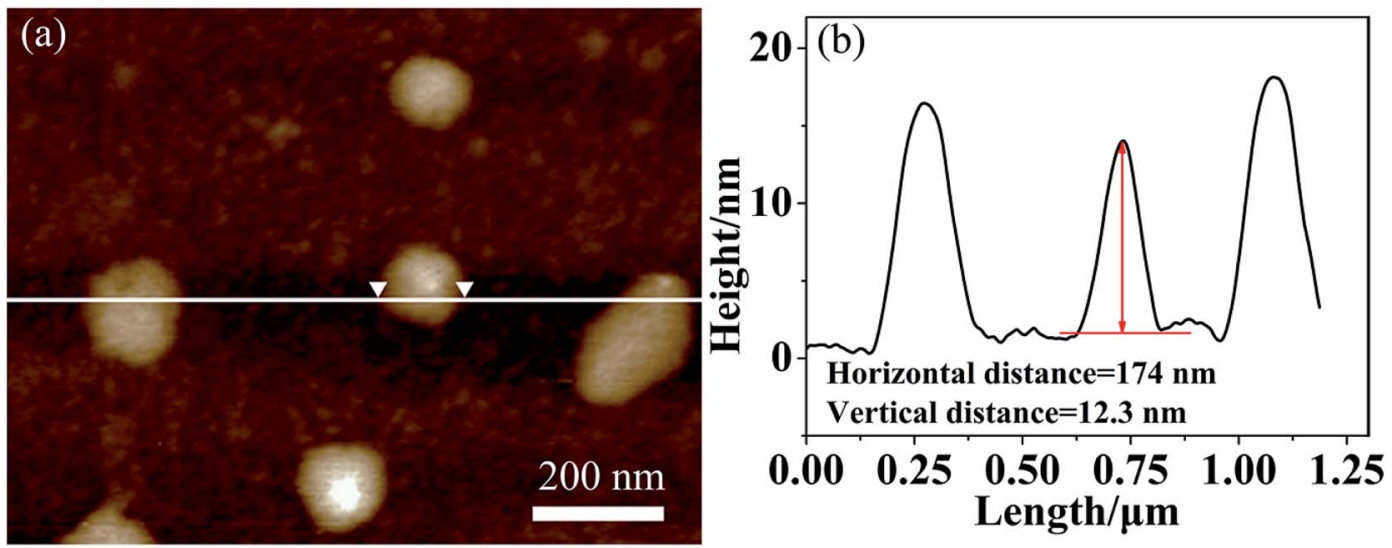

Fig. 4 Tapping-mode AFM image (a) and section analysis profile (b) of multicompartment-like aggregates.

during the sample drying process. The far larger diameter/ height ratio of about 15 reflects the flexible and soft-binding property of the alkyl chain corona and the partial collapse of compartments onto the solid surface after solvent evaporation, both of which should further lead to a flattened morphology of the aggregates. ${ }^{23}$

The multilayered wall structure of the aggregate was confirmed by XRD analysis. As can be seen from a typical result for a solvent-free sample shown in Fig. 5a, several diffraction peaks could be the indexed characteristic for a lamellar arrangement. The three Bragg diffraction peaks could be observed at $3.08^{\circ}, 6.21^{\circ}$ and $7.94^{\circ}$, corresponding to the layer thickness of $2.87,1.41$ and $1.01 \mathrm{~nm}$. The value of $2.87 \mathrm{~nm}$ was consistent with that $(2.90 \mathrm{~nm})$ mapped for the repeat layer spacing under the HR-TEM. According to the model reported in literatures, ${ }^{23-25}$ such a multilayered structure could be reasonably recognized as an parallel packed inverse SEC layers with POMs located in the middle while the BFDMA molecules located on both sides of the POM.

To confirm such an inverse layered packing of SEC, the optimized geometry of BFDMA was explored using the density functional theory (DFT) calculation carried out with a mixed basis set of LANL2DZ for Fe, and 6-31 G(d, p) for C, H and N atoms,${ }^{49}$ which was applied to evaluate the length of alkyl chain of single BFDMA molecule. All calculations were performed at the B3LYP level using the Gaussian 09 package. Fig. 5b shows the optimized geometries of this double chain surfactant with the length of about $1.71 \mathrm{~nm}$ for one chain including the quaternary ammonium headgroup and ferrocene group. Based on this result and the diameter of a single Keggin-type POM cluster (ca. $1.0 \mathrm{~nm}),{ }^{50}$ the ideal total thickness of a single layer of SEC with BFDMA in both sides could be estimated to be $4.42 \mathrm{~nm}$, which is larger than the $d$-spacing obtained by XRD $(2.87 \mathrm{~nm})$. Therefore, an evidently interdigitated SEC layers was much probable for the observed wall structure, where the alkyl chains of BFDMA were interdigitated and packed in a high order with the ferrocene rings stacked each other.

\subsection{Mechanism for multicompartment-like aggregate formation}

To get a reasonable aggregation mechanism for above mentioned morphology evolution, the molecular packing of

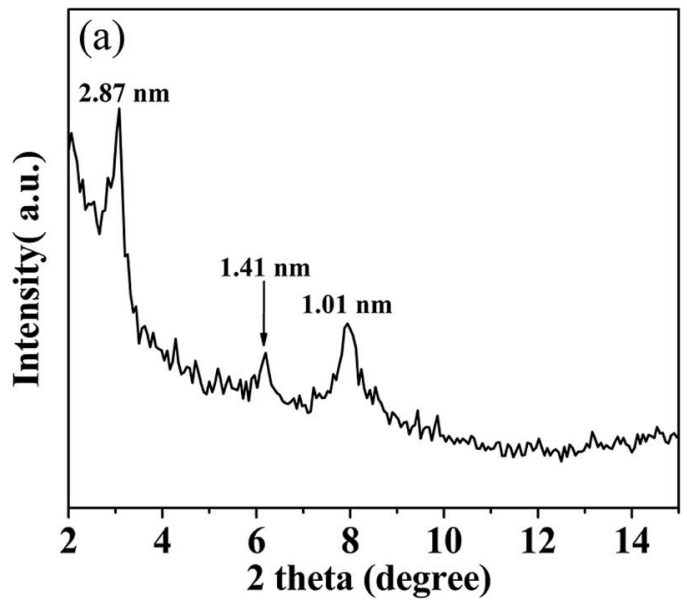

(b)

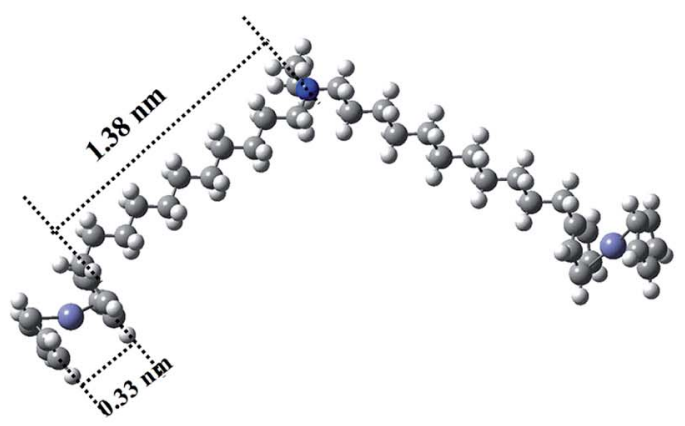

Fig. 5 (a) XRD pattern of SEC aggregates. (b) Geometries of BFDMA molecule optimized using the model at the B3LYP/6-31G(d, p) level. The gray, blue, white and purple balls represent carbon, nitrogen, hydrogen and iron atoms, respectively. 
BFDMA molecules on the surface of POM and the possible rearrangement during aggregation should be clarified. As well known, the Keggin structure of POM is composed of a $\mathrm{PO}_{4}{ }^{3-}$ unit as heteroatom core and twelve $\mathrm{WO}_{6}(\mathrm{M}=\mathrm{Mo}, \mathrm{W}$, etc. $)$ octahedrons as the shell, in which their edges and corners are shared to create a cage shape. ${ }^{23}$ Therefore, when the SEC building block was formed, three BFDMA molecules were electrostatically drawn by the central $\mathrm{PO}_{4}{ }^{3-}$ but separated by the $\mathrm{W}_{12} \mathrm{O}_{36}$ cage (Fig. 6A). Such a soft binding model provides the possibility for surfactant molecules to reorganize on the $\mathrm{W}_{12} \mathrm{O}_{36}$ surface corresponding to the particular environment condition change to achieve to the new thermodynamic equilibrium between non-covalent interactions. ${ }^{51}$ As shown in Fig. 6, in the mixed solvent here, the surfactant molecules on the POM surface adopted an asymmetrically distribution during the aggregation with two BFDMA on one side while another BFDMA on the other side to produce a curved layer (Fig. 6B). Both the strong solvophobic effect among the alkyl chains and the nearly rod like building block shape induced the formation of vesicleslike aggregate with multilayered shells (Fig. 6C). The vesicle wall was composed of one POM monolayer mediated surfactant bilayers with POM clusters inside and BFDMA chains outside, which has also been reported previously. ${ }^{23,51}$ However, these aggregates exhibited different characters compared to those fabricated by conventional surfactants capped polyoxometalate where the spherical, nanotube-like and other morphologies could keep stable. In our situation, the obtained spherical hollow aggregates tended to coalesce to each other with time. During this process, the wall fusion of adjacent outside vesicles occurred to form a shell, while the vesicles inside kept stable (Fig. 6D). We assumed that the wall fusion also occurred only mildly inside the aggregates. The outermost BFDMA layers containing ferrocene groups could easily contact with each other and the solvophobic effect between alkyl chains and $\pi-\pi$ interaction between ferrocene groups would both enhance such fusion. However, the POMs sandwiched in the middle of SEC layers might hinder the further fusion between the inner alkyl chains. Then, the multicompartment-like aggregates with thin shell were finally obtained. It is interesting that such a tendency was more obvious in our study due to the capping molecule structure: the alkyl chains containing ferrocene groups rearranged to minimize the interfacial energy and tension between the hydrophobic parts with the solvent, and therefore the fusion of wall structure taken place. ${ }^{52}$

\subsection{Redox responsibility of multicompartment aggregates}

The reversibility of such redox-responsive self-assemblies of SEC was also investigated preliminarily by measuring their cyclic voltammetry (CV) behaviors with the results shown in Fig. 7. The redox potentials of pure ferrocene sample were firstly measured as the internal standard (inset of Fig. 7a). The CV profiles of individual BFDMA and SEC dissolved in $0.1 \mathrm{M}$ tetrabutylammonium perchlorate (TBAP) at concentrations of 0.24

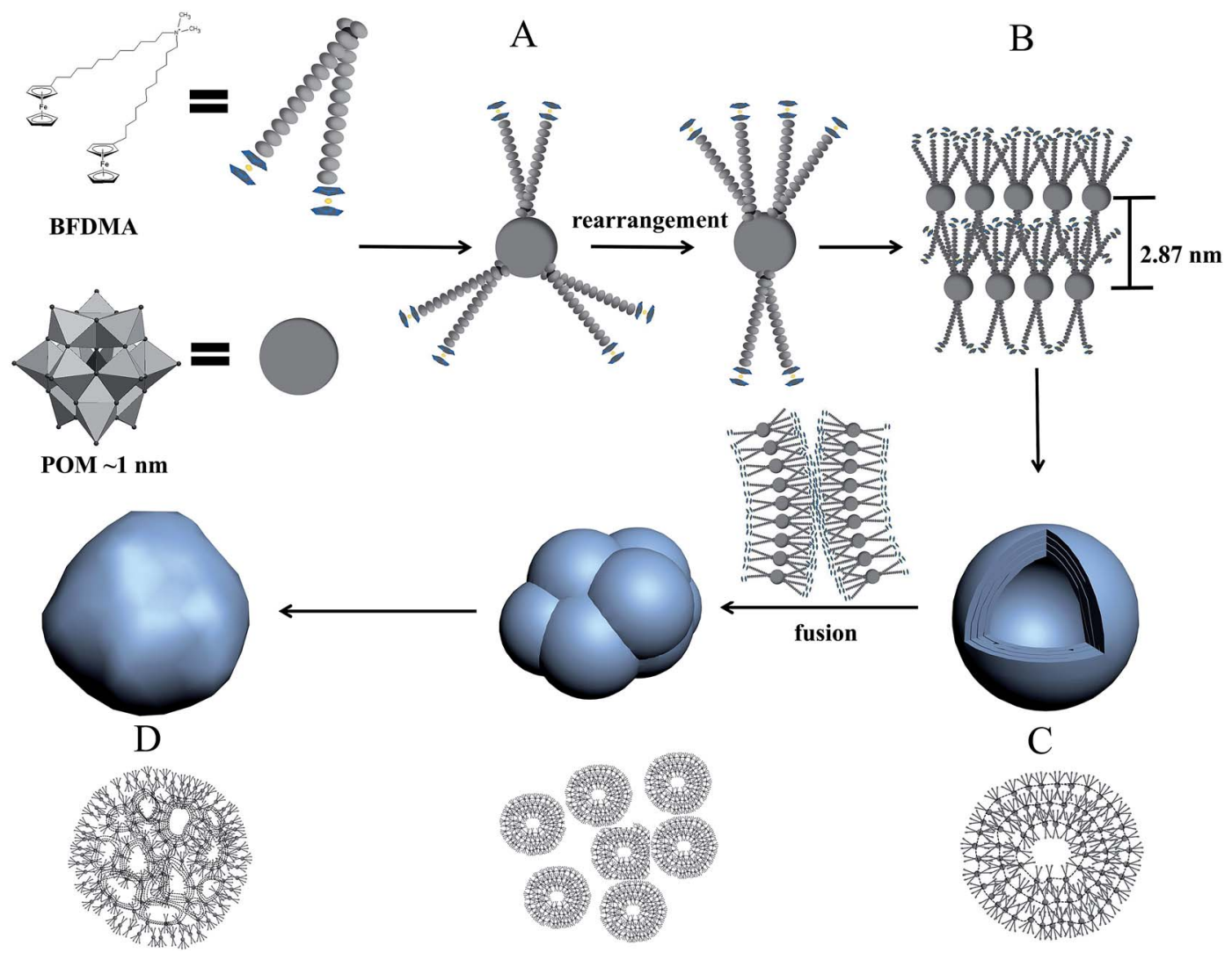

Fig. 6 Assembling mechanism of (BFDMA) ${ }_{3} \mathrm{PW}_{12} \mathrm{O}_{40}$ in DMF/butanol solution, including initial formation of SEC (A), rearrangement of $\mathrm{BFDMA}$ (B), inversed-vesicle assembly (C) and fusion of the outer walls to form multicompartment-like aggregates (D). 

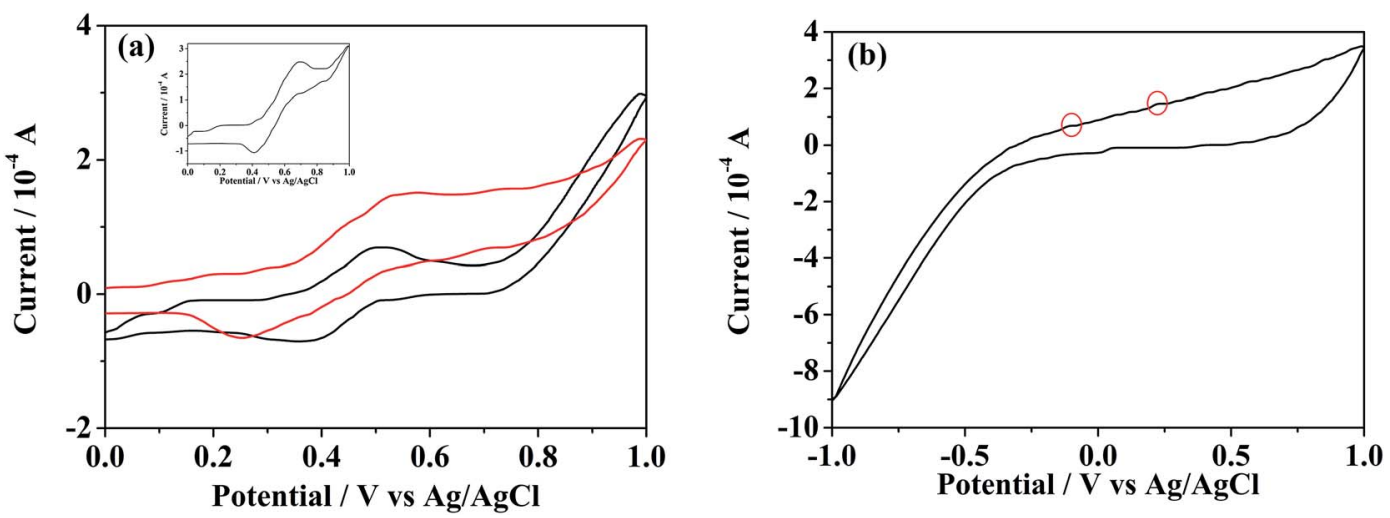

Fig. 7 Cyclic voltammograms of BFDMA and SEC solution with inset for ferrocene as internal standard (a) and individual POM solution (b) in DMF/ butanol $\left(\mathrm{v} / \mathrm{v}=3:\right.$ 7) plus $0.1 \mathrm{M}$ TBAP at a scan rate of $0.1 \mathrm{~V} \mathrm{~s}^{-1}$.

and $0.53 \mathrm{mg} \mathrm{mL}^{-1}$ respectively (keep the same concentration of ferrocene groups) are shown in Fig. 7a. Similar CV profiles to that of pure ferrocene could be seen. The half-wave peak of BFDMA solution was about $0.436 \mathrm{~V}$, while that in SEC solution exhibited as $0.397 \mathrm{~V}$. Such a shift of the redox peak for SEC compared to BFDMA might be influenced by POM cluster unit. The CV profile shown in Fig. $7 \mathrm{~b}$ was from POM solution $\left(0.53 \mathrm{mg} \mathrm{mL}{ }^{-1}\right)$, where the redox peaks were not readily visible and there were only two very tiny oxidized peaks (denoted by red circles) and a broad reduced peak. It was likely that the obviously electrochemical signals of POM occurred at the range beyond -1.0 to $+1.0 \mathrm{~V}(v s$. $\mathrm{Ag} / \mathrm{AgCl})$. However, the more obvious peaks associated with solvent breakdown might obscure the POM signals if applied more negative or positive potentials than $\pm 1.0 \mathrm{~V}$ in our conditions, which was in much similar case to that as described by Cronin..$^{53}$

To certify the supposed aggregation mechanism and test if the SEC aggregates were well redox-responsive, a potentiostatic method was carried out for SEC self-assembling system at a concentration of $0.53 \mathrm{mg} \mathrm{mL}$. Upon applied an anodic potential $(+0.81 \mathrm{~V} v s$. $\mathrm{Ag} / \mathrm{AgCl})$ larger than the peak potential of ferrocene oxidation for $4 \mathrm{~h}$, the ferrocene groups at the terminal of SEC was oxidized into positively charged ferrocenium as confirmed by the UV-vis absorption results shown in Fig. 8a. The absorption peak at about $430 \mathrm{~nm}$ corresponding to $\pi-\pi^{*}$ electron transition of ferrocene group in reduced state disappeared almost after oxidation. Then, the decrease of solvophobic parts of SEC could cause the instability of aggregates and accelerate the disassembly of initially formed aggregates. Meanwhile, the applied anodic potential might also reduce the negative charges of POM. Both effects would synergistically destroy the original encapsulation by BFDMA and therefore the SEC block, which resulted in the disassembly of the multicompartment-like aggregate.

The corresponding TEM image for these disassembled aggregates shown in Fig. 8b indicated that they still presented the spherical morphology but with at a larger size of $c a .200 \mathrm{~nm}$. These spherical aggregates resulted from the pseudo-bola amphiphilic molecules due to formation of solvophilic ferrocenium at the terminal of BFDMA chain..$^{54,55}$ These newly produced solvophilic parts might swell more in solution and cause the aggregate size slightly increased. ${ }^{56}$ On the other hand, the positively charged ferrocenium might increase the electrostatic repulsion between chains and lead to an incompact arrangement of the outside alkyl chains, which might also induce larger size aggregates. The reversibility of such redox-responsive self-
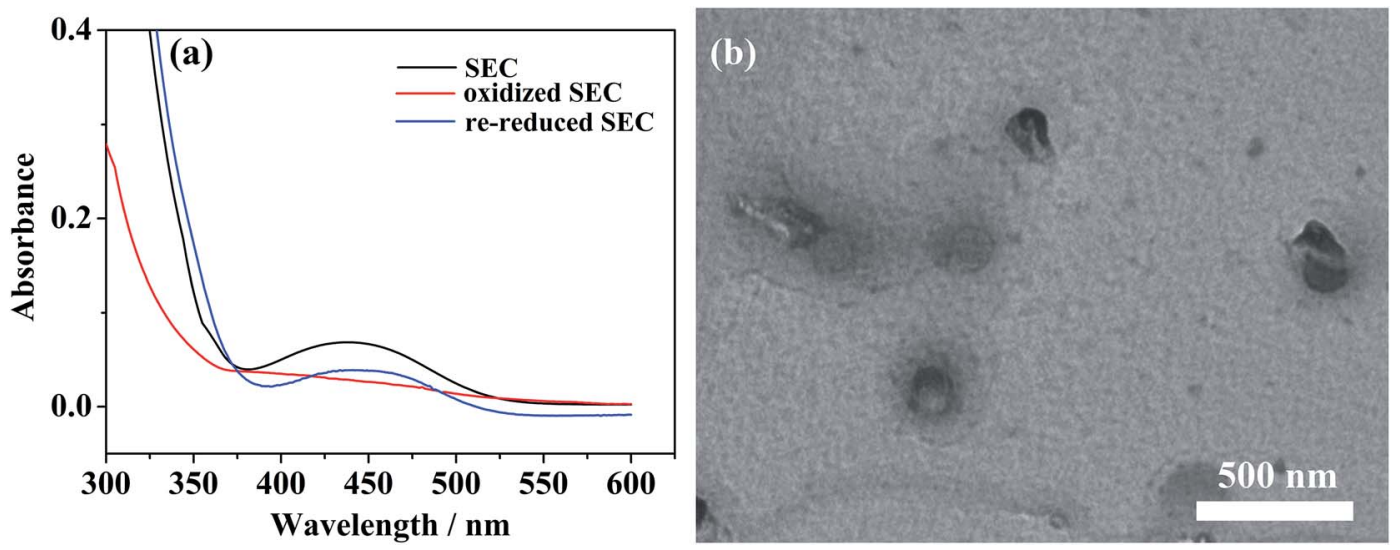

Fig. 8 (a) UV/vis spectra of SEC solutions in different redox states; (b) TEM image of disassembled aggregates after oxidation of ferrocene groups. 
assemblies was also explored. After a cathodic reduction potential $(-0.81 \mathrm{~V} v s$. $\mathrm{Ag} / \mathrm{AgCl})$ was applied for $4 \mathrm{~h}$, the absorption band at about $434 \mathrm{~nm}$ in UV-vis profile for re-reduced SEC in Fig. 8a could be again noted. The comparison of TEM images of multicompartment-like aggregates formed initially and restored was shown in Fig. S4. $\dagger$ The decreased number of the restored aggregates implied a limited reversibility of this system.

\section{Conclusions}

In conclusion, the surfactant encapsulated complex composed of a Keggin-type POM and three BFDMA molecules containing redox-active ferrocene groups has been constructed through electrostatic interaction. Such supra-amphiphilic building blocks could be assembled into hollow spherical aggregates with multilayered shells in DMF/butanol mixed organic solvent at initial growth stage, which was promoted by the solvophobic effect between the alkyl chains of surfactant and their organized rearrangement around the exterior of the POM cluster. The unique inverse wall structure with ferrocene groups outside made it easy for these aggregates to fuse their outermost shell to form multicompartment-like aggregates with increasing aging time. Such a fusion was mainly driven by solvophobic effect and also by $\pi-\pi$ stacking between ferrocene groups. These aggregates were stable both in solution and in dried status. However, applying an anodic oxidation potential, the multicompartmentlike aggregates were disassembled to form larger spherical aggregates due to the reduction of solvophobic effect by the additional electrostatic repulsion among newly produced positively charged ferroceniums. To recover the multicompartmentlike aggregates, we just need to switch the applied electrochemical stimulus to a cathodic reduction potential. The obtained results here should be referable for designing novel POM-based nanostructures. Meanwhile, such inorganic/ organic hybrid induced compartment-like hollow aggregates may play as vehicles for material transportation or act as a host for metal nanocrystals preparation, which should highly extend their applications in the fields of catalysis or drug delivery.

\section{Acknowledgements}

We are thankful for the financial supports from the National Natural Science Foundation of China (21373127 and 21673129).

\section{References}

1 Y. Wang, N. Ma, Z. Wang and X. Zhang, Angew. Chem., Int. Ed., 2007, 46, 2823-2826.

2 H. Goto, H. Q. Zhang and E. Yashima, J. Am. Chem. Soc., 2003, 125, 2516-2523.

3 J. Wang, W. L. Cui, S. J. Yi and X. Chen, Colloids Surf., A, 2015, 484, 28-36.

4 S. Aikawa, R. G. Shrestha, T. Ohmori, Y. Fukukita, Y. Tezuka, T. Endo, K. Torigoe, K. Tsuchiya, K. Sakamoto, K. Sakai, M. Abe and H. Sakai, Langmuir, 2013, 29, 5668-5676.

5 M. Motornov, Y. Roiter, I. Tokarev and S. Minko, Prog. Polym. Sci., 2010, 35, 174-211.
6 S. Mura, J. Nicolas and P. Couvreur, Nat. Mater., 2013, 12, 991-1003.

7 X. Zhang and C. Wang, Chem. Soc. Rev., 2011, 40, 94-101.

8 B. Rybtchinski, ACS Nano, 2011, 5, 6791-6818.

9 L. J. Prins, D. N. Reinhoudt and P. Timmerman, Angew. Chem., Int. Ed., 2001, 40, 2382-2426.

10 F. J. Xiao, C. X. Yin, E. X. Wang and Y. J. Luo, J. Inorg. Organomet. Polym., 2010, 20, 278-283.

11 T. Daeneke, T. H. Kwon, A. B. Holmes, N. W. Duffy, U. Bach and L. Spiccia, Nat. Chem., 2011, 3, 211-215.

12 B. Donnio, J. M. Seddon and R. Deschenaux, Organometallics, 2000, 19, 3077-3081.

13 N. Sato and H. Okuma, Sens. Actuators, B, 2008, 129, 188194.

14 X. Y. Liu and N. L. Abbott, J. Colloid Interface Sci., 2009, 339, 1-18.

15 A. Dolbecq, E. Dumas, C. R. Mayer and P. Mialane, Chem. Rev., 2010, 110, 6009-6048.

16 A. Proust, R. Thouvenot and P. Gouzerh, Chem. Commun., 2008, 1837-1852.

17 E. Coronado, C. Gimenez-Saiz and C. J. Gomez-Garcia, Coord. Chem. Rev., 2005, 249, 1776-1796.

18 J. M. Clemente-Juan, E. Coronado and A. Gaita-Arino, Chem. Soc. Rev., 2012, 41, 7464-7478.

19 C. L. Hill, Chem. Rev., 1998, 98, 1.

20 D. Barats and R. Neumann, Adv. Synth. Catal., 2010, 352, 293-298.

21 T. Zhang, J. Brown, R. J. Oakley and C. F. J. Faul, Curr. Opin. Colloid Interface Sci., 2009, 14, 62-70.

22 M. M. Farahani, J. Movassagh, F. Taghavi, P. Eghbali and F. Salimi, Chem. Eng. J., 2012, 184, 342-346.

23 H. L. Li, H. Sun, W. Qi, M. Xu and L. X. Wu, Angew. Chem., Int. Ed., 2007, 46, 1300-1303.

24 A. Nisar, J. Zhuang and X. Wang, Chem. Mater., 2009, 21, 3745-3751.

25 A. Nisar, X. X. Xu, S. L. Shen, S. Hu and X. Wang, Adv. Funct. Mater., 2009, 19, 860-865.

26 Y. F. Song, N. McMilla, D. L. Long, J. Thiel, Y. Ding, H. Chen, N. Gadegaard and L. Cronin, Chem.-Eur. J., 2008, 14, 23492354.

27 J. Zhang, Y. F. Song, L. Cronin and T. Liu, J. Am. Chem. Soc., 2008, 130, 14408-14409.

28 J. Zhang, Y. F. Song, L. Cronin and T. Liu, Chem.-Eur. J., 2010, 16, 11320-11324.

29 T. R. Zhang, S. Q. Liu, D. G. Kurth and C. F. J. Faul, Adv. Funct. Mater., 2009, 19, 642-652.

30 W. Bu, H. Fan, L. Wu, X. Hou, C. Hu, G. Zhang and X. Zhang, Langmuir, 2002, 18, 6398-6403.

31 A. Nisar, Y. Lu and X. Wang, Chem. Mater., 2010, 22, 35113518.

32 Y. Yan, H. B. Wang, B. Li, G. F. Hou, Z. D. Yin, L. X. Wu and V. W. W. Yam, Angew. Chem., Int. Ed., 2010, 122, 9233-9236.

33 Y. Yan, B. Li, Q. Y. He, Z. F. He, H. Ai, H. B. Wang, Z. D. Yin and L. X. Wu, Soft Matter, 2012, 8, 1593-1600.

34 P. L. Veya and J. K. Kochi, J. Organomet. Chem., 1995, 488, C4-C8. 
35 Y. J. Niu, X. Y. Ren, B. Yin, D. J. Wang, G. L. Xue, H. M. Hu, F. Fu and J. W. Wang, J. Organomet. Chem., 2010, 695, 18631868.

36 J. L. Stark, V. G. Young and E. A. Maatta, Angew. Chem., Int. Ed., 1995, 34, 2547-2548.

37 N. Yoshino, H. Shoji, Y. Kondo, Y. Kakizawa, H. Sakai and M. Abe, J. Jpn. Oil Chem. Soc., 1996, 45, 769-789.

38 H. L. Li, Y. Yang, W. Li, L. H. Bi and L. X. Wu, Chem. Commun., 2010, 46, 3750-3752.

39 J. C. Yu, A. W. Xu, L. Z. Zhang, R. Q. Song and L. X. Wu, J. Phys. Chem. B, 2004, 108, 64-70.

40 Y. X. Guo, Y. J. Gong, Z. D. Yu, Y. A. Gao and L. Yu, RSC Adv., 2016, 6, 14468-14473.

41 T. Rajkumar and G. R. Rao, J. Chem. Sci., 2008, 120, 587-594.

42 H. S. Xu, L. Zhuang, Z. X. Li, Z. F. Li, H. M. Hu and G. L. Xue, J. Cluster Sci., 2010, 21, 211-221.

43 J. Umemura, D. G. Cameron and H. H. Mantsch, Biochim. Biophys. Acta, 1980, 602, 32-44.

44 H. Sapper, D. G. Cameron and H. H. Mantsch, Can. J. Chem., 1981, 59, 2543-2549.
45 D. A. Myrzakozha, T. Hasegawa, J. Nishijo, T. Imae and Y. Ozaki, Langmuir, 1999, 15, 6890-6896.

46 W. F. Bu, H. L. Li, W. Li, L. X. Wu, C. X. Zhai and Y. Q. Wu, J. Phys. Chem. B, 2004, 108, 12776-12782.

47 C. Wang, Y. T. Kang, K. Liu, Z. B. Li, Z. Q. Wang and X. Zhang, Polym. Chem., 2012, 3, 3056-3059.

48 A. H. Gröschel and A. H. E. Müller, Nanoscale, 2015, 7, 11841-11876.

49 G. Huang and P. Liu, ACS Catal., 2016, 6, 809-820.

50 D. Long, E. Burkholder and L. Cronin, Chem. Soc. Rev., 2007, 36, 105-121.

51 A. Nisar and X. Wang, Dalton Trans., 2012, 41, 9832-9845.

52 P. Shi, Y. Qu, C. Liu, H. Khan, P. Sun and W. Zhang, ACS Macro Lett., 2015, 5, 88-93.

53 J. Thiel, P. I. Molina, M. D. Symes and L. Cronin, Cryst. Growth Des., 2012, 12, 902-908.

54 R. Nagarajan, Chem. Eng. Commun., 1987, 55, 251-273.

55 G. D. Wang, C. Wang, Z. Q. Wang and X. Zhang, Langmuir, 2011, 27, 12375-12380.

56 L. Liu, L. Rui, Y. Gao and W. Zhang, Polym. Chem., 2015, 6, 1817-1829. 